\title{
POINT/COUNTERPOINT
}

Suggestions for topics suitable for these Point/Counterpoint debates should be addressed to the Moderator: William R. Hendee, Medical College of Wisconsin, Milwaukee: whendee@mcw.edu. Persons participating in Point/Counterpoint discussions are selected for their knowledge and communicative skill. Their positions for or against a proposition may or may not reflect their personal opinions or the positions of their employers.

\section{Diagnostic ultrasound should be performed without upper intensity limits}

\author{
William D. O’Brien, Jr. \\ University of Illinois at Urbana-Champaign, Department of Electrical \& Computer Engineering, \\ Bioacoustic Research Lab, Urbana, Illinois 61801 (Tel: 217/333-2407,E-mail:wdo@uiuc.edu) \\ Douglas Miller \\ University of Michigan, Department of Radiology, Ann Arbor, Michigan (Tel: 734/647-3344, \\ E-mail:douglm@umich.edu)
}

William R. Hendee, Moderator

(Received 13 October 2000; accepted for publication 13 October 2000)

[DOI: 10.1118/1.1335500]

\section{OVERVIEW}

As with most diagnostic technologies, ultrasound imaging reflects a trade-off between image resolution and energy absorption in tissue. With diagnostic ultrasound, current upper limits on beam intensity have not been correlated with demonstrated harmful effects. Microcavitation has been observed at intensities near these limits, but its biological significance is unknown. This Point/Counterpoint explores whether upper intensity limits should be removed to permit improvements in the quality of ultrasound images.

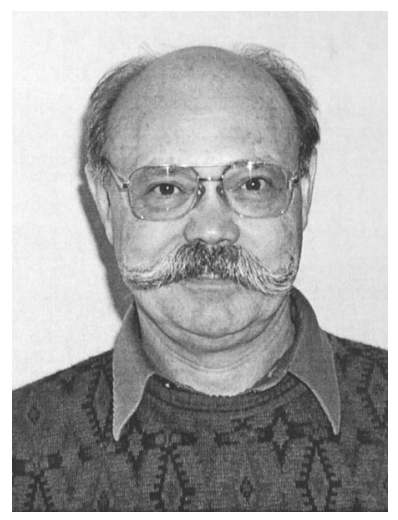

Arguing for the Proposition is William D. O'Brien, Jr., Ph.D. Dr. O'Brien is Professor of Electrical and Computer Engineering and of Bioengineering, College of Engineering; Professor of Bioengineering, College of Medicine; and Director of the Bioacoustics Research Laboratory at the University of Illinois. Previously, he worked at the Bureau of Radiological Health (currently the Center for Devices and Radiological Health) of the U.S. Food and Drug Administration. He is a fellow of four professional societies; has served as president of the IEEE Ultrasonics, Ferroelectrics, and Frequency Control Society and the American Institute of Ultrasound in Medicine; and is Editor-in-Chief of the IEEE Transactions on Ultrasonics, Ferroelectrics, and Frequency Control. His research interests involve the many areas of ultrasoundtissue interaction, including spectroscopy, risk assessment, biological effects, tissue characterization, dosimetry, and imaging for which he has published 215 papers.

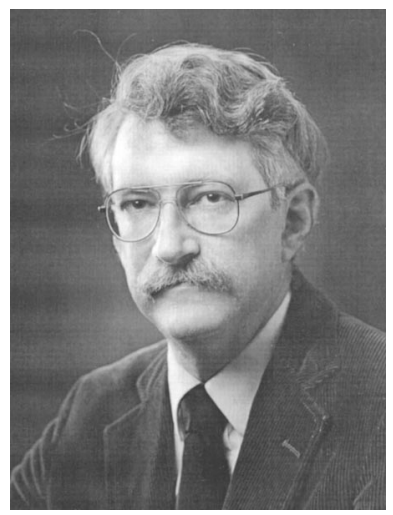

Arguing against the Proposition is Douglas Miller, Ph.D. Dr. Miller is a Senior Research Scientist at the University of Michigan Department of Radiology. He received a Ph.D. in Physics from the University of Vermont in 1976, and worked at Battelle Pacific Northwest National Laboratory on bioelectromagnetics and ultrasonic biophysics research before moving to Michigan. Dr. Miller has served on ultrasound safety review groups of the American Institute of Ultrasound in Medicine, World Federation of Ultrasound in Medicine and the National Council on Radiation Protection and Measurements. Present NIH projects include research on the bioeffects associated with contrast aided diagnostic ultrasound and on ultrasound enhanced cancer gene therapy.

\section{FOR THE PROPOSITION: William D. O'Brien, Jr.,} Ph.D.

\section{Opening Statement}

Regulatory control of diagnostic ultrasound equipment in the U.S. can be traced to passage of the 1976 Medical Device Amendments to the Food, Drug, and Cosmetic Act. When the FDA initiated the regulation of diagnostic ultrasound equipment in its 1985 " $510(\mathrm{k})$ premarket notification,", application-specific intensity limits were set that manufactur- 
ers could not exceed. The 510(k)'s purpose was for the FDA to assess if a new device was "substantially equivalent," in safety and effectiveness, to diagnostic ultrasound equipment on the market prior to 1976 . However, the intensity limits were not based on safety or effectiveness but rather on the maximum intensity limits of diagnostic ultrasound equipment at the time when the Amendments were enacted, in 1976; hence the term pre-amendments levels. To emphasize the FDA's date-based regulatory approach, as opposed to safety and efficacy based, the American Institute of Ultrasound in Medicine notified the FDA in mid-1986 that there existed prior to May 28, 1976 at least two diagnostic ultrasound devices with intensity levels greater than the 1985 application-specific intensity limits. In early 1987, the FDA updated their limits to the higher intensity levels.

Following widespread approval of the voluntary Output Display Standard (ODS) in early 1990, the FDA essentially adopted the ODS for its regulatory guidelines. The ODS did not include upper limits. Nevertheless, the FDA added application-nonspecific guideline upper limits that were still based on the 1976 pre-amendments levels.

Problems with the date-based upper-limit regulatory approach include (1) a complicated set of rules and procedures by which manufacturers verify to the FDA that their equipment is in compliance, and the costs associated with these requirements; (2) a perception that these upper limits are safe; (3) a demonstrated lack of attention to ODS education materials about the safety-based biophysical indicators; (4) the exposure of patients at these upper limits for which there may be safety concerns; (5) a limiting of future clinical benefits by preventing the development of more advanced diagnostic ultrasound systems at higher levels; and, finally, (6) a recognition that limiting diagnostic ultrasound capabilities may, in fact, be responsible for greater patient risk due to either an inadequate diagnosis, or to the use of an additional diagnostic procedure for which there is a defined risk.

The elimination of the upper-limit regulatory approach would have the following benefits: (1) a less complicated set of rules and procedures by manufacturers, and at less cost; (2) the elimination of the perception that there are safe limits; (3) more attention to the ODS education materials; (4) more attention to the ODS-based biophysical indicators; (5) making available research opportunities to develop advanced diagnostic procedures; and (6) providing the diagnostic capability to obtain an adequate diagnosis if higher levels are required.

To apply rigid controls to ultrasound intensity without a proper scientific justification benefits no one, particularly the patient. The physician is a professional trained to provide health care by making informed benefit-risk judgements. The FDA's regulatory approach had denied the physician the need to become informed about such benefit-risk issues, and for that we are all worse off.

\section{Rebuttal}

The current government-mandated upper-intensity-limit regulatory approach has placed the risk side of the risk- benefit decision on the FDA, not with physicians trained to make such decisions. This is not how good medicine should be practiced. In an ideal world, the government would protect us. The government consists of individuals like you and me, and none of us have the knowledge or wisdom to know how to provide long-distance protection. Protection goes well beyond making sure that diagnostic devices do not produce any bioeffects. Protection must include on-the-scene decision making. In other words, a fundamental clinical issue is an accurate and safe diagnosis of the patient. That is why physicians receive extensive training in the risk-benefit decision making process.

My opponent argues that risk can be eliminated by limiting outputs to values below the threshold. Obviously, this refers to ultrasound-induced risk. What about the risk associated with an insufficient diagnostic quality image? What about the follow-on diagnostic procedure that might have a significant hazard? We cannot view risk narrowly. It must be viewed in the broadest sense, that of providing the best diagnosis of the patient.

My opponent also argues that limits provide a conceptual separation between diagnostic and therapeutic uses of ultrasound, and that built-in safety limits free the operator from basic issues such as safety, equipment operation, and complex dose calculations. Arbitrary boundaries between types of equipment are artificial, but operator training is not. Users must have appropriate training. The safety issue must be argued on what is best for the patient's health.

My opponent finally argues that without limits, manufacturers would be prone to engage in competition for higher power for anticipated marginal increases in image quality. Even if these were true, and this is unsupported, the marginal increase in image quality might make the diagnostic difference for some patients. Is this not worth it? Should we not try it?

\section{AGAINST THE PROPOSITION: Douglas Miller, Ph.D. \\ Opening Statement}

The use of upper limits appears to be an ideal way to promote the safety of diagnostic ultrasound examinations. Bioeffects of ultrasound occur by way of indirect mechanisms, such as heating or acoustical cavitation, and appear to have identifiable thresholds. The risk of such an effect can be eliminated by limiting outputs to values below the threshold. Of course, the exact forms and values of upper limits on instrument output should be continually questioned and improved. The present guideline limits ${ }^{1}$ are arbitrarily linked to the state of the art in 1976, rather than to scientific principles.

However, through deliberation and consensus within the ultrasound community, the restrictive limits originally applied to many examinations have been raised to the benefit of effective diagnosis. In addition, on-screen readouts of thermal and mechanical exposure indices provided on many recent systems are valuable for the identification and management of worse-case conditions, for which some potential for bioeffects exists with current machines both from heating 
(most likely of bone) and from mechanical effects (most likely on lung or intestine).

The use of well-chosen upper limit guidelines for diagnostic ultrasound also has several other benefits. Limits provide a conceptual separation of diagnostic and therapeutic uses of ultrasound, thus delineating regimes for patient safety on the one hand, and for effective treatment (e.g., physical therapy, surgery, or lithotripsy) on the other hand. Simple built-in safety limits free the operator from complex dose calculations, detailed risk/benefit determinations or extensive safety training for use of an instrument. Furthermore, authoritative upper limits engender a degree of public confidence in the safety of examinations not possible without them.

Eliminating upper limit guidelines is a bad idea for several reasons. If this were done, instrument manufacturers would be prone to engage in competition for higher power for anticipated marginal increases in image quality (e.g., a doubling of intensity only provides an additional $6 \%$ imaging depth, even for linear propagation ${ }^{2}$ ). Alternately, higher output power might be substituted for expensive receiver amplifiers, thus increasing risk with no real patient benefit. Most importantly, it seems likely that patient harm would eventually be encountered.

In summary, guideline upper limits for diagnostic ultrasound have many advantages, and even the present imperfect limits have served manufacturers, physicians and patients well. Eliminating limits, which would likely be of only marginal value for improving image quality, would also eliminate the confidence of the medical community and the general public in the safety of diagnostic ultrasound examinations. Diagnostic ultrasound should not be performed without upper intensity limits.

\section{Rebuttal}

Dr. O'Brien has clearly stated the problems perceived to be associated with the existence of guideline upper limits for diagnostic ultrasound. However, removing the present limits would not solve the problems he has listed because (1) manufacturers would still have to follow complicated procedures in order to provide the output display indices for FDA clearance; (2) the present limits are justified, to some extent, by extensive research and through considerations of bioeffects mechanisms; (3) the elimination of limits would do little to advance knowledge or education about safety issues; (4) safety concerns related to present outputs would certainly not be ameliorated by the removal of upper limits; (5) in fact, higher levels are not prohibited, and a truly compelling new device requiring levels in excess of the guidelines can gain FDA approval by demonstrating safety and efficacy; (6) greater advances in diagnostic ultrasound would be obtained by improving training in imaging procedures, safety issues, and diagnostic interpretation than by slight improvements in images at higher intensities.

The removal of guideline limits would permit manufacturers to design diagnostic ultrasound instruments to engineering limits regardless of safety issues, even though the engineering objective may not serve more general medical needs and desires. Physicians and sonographers should focus on the art of sonography and diagnosis rather than on complex safety issues related to the selection of an intensity for each examination. Many patients do not receive any discernible benefit from a diagnostic test, and consequently expect to be protected from unnecessary levels of risk. The present upper limit guidelines, though less than perfect, satisfy these general needs and work well in the real world of incomplete safety information and inadequate education. Manufacturers, sonographers, and patients all benefit from the framework provided by the existing approval process. Guideline upper limits for diagnostic ultrasound should be continually improved, but should not be removed.

${ }^{1}$ FDA, "Information for manufacturers seeking clearance of diagnostic ultrasound systems and transducers." CDRH, U. S. Food and Drug Administration, Rockville, MD, 1997.

${ }^{2}$ NCRP, "Exposure criteria for medical diagnostic ultrasound: I. Criteria based on thermal mechanisms." National Council on Radiation Protection and Measurement, Bethesda, MD, 1992. 